\title{
Author Correction: Two million years of flaking stone and the evolutionary efficiency of stone tool technology
}

\author{
Željko Režek (D), Harold L. Dibble, Shannon P. McPherron (D), David R. Braun and Sam C. Lin
}

Correction to: Nature Ecology \& Evolution https://doi.org/10.1038/s41559-018-0488-4, published online 5 March 2018.

In the version of this Article originally published, the authors mistakenly included duplicate entries in the flake datasets for the new Pech de l'Azé IV and Warwasi collections, resulting in minor errors in the statistical analysis. The authors have now repeated this analysis with the correct flake datasets. As a result, in the following two sentences, the number of flakes has been changed from 19,000 to 18,000: "Using more than 18,000 flakes from 81 assemblages spanning two million years..." and "We applied a comparative approach...on more that 18,000 complete and unmodified flakes." In addition, in Figs. 1-3 and Supplementary Fig. 1, some of the data points for the Pech de l'Azé IV and Warwasi collections have moved; the original and corrected figures are below. Supplementary Tables 1 and 2 have been updated to reflect the corrected statistics, and datasets 'Flake_data' and 'Summary_data' have been replaced with the corrected data files. Furthermore, the data availability statement has been updated with the text "Open access to these data and the R code generated for this study is provided at https://zenodo.org/record/1408081\#.W6iyn84zaHs". The authors would like to thank L. Premo at Washington State University for finding the duplicate entries in the published flake dataset. 
Original

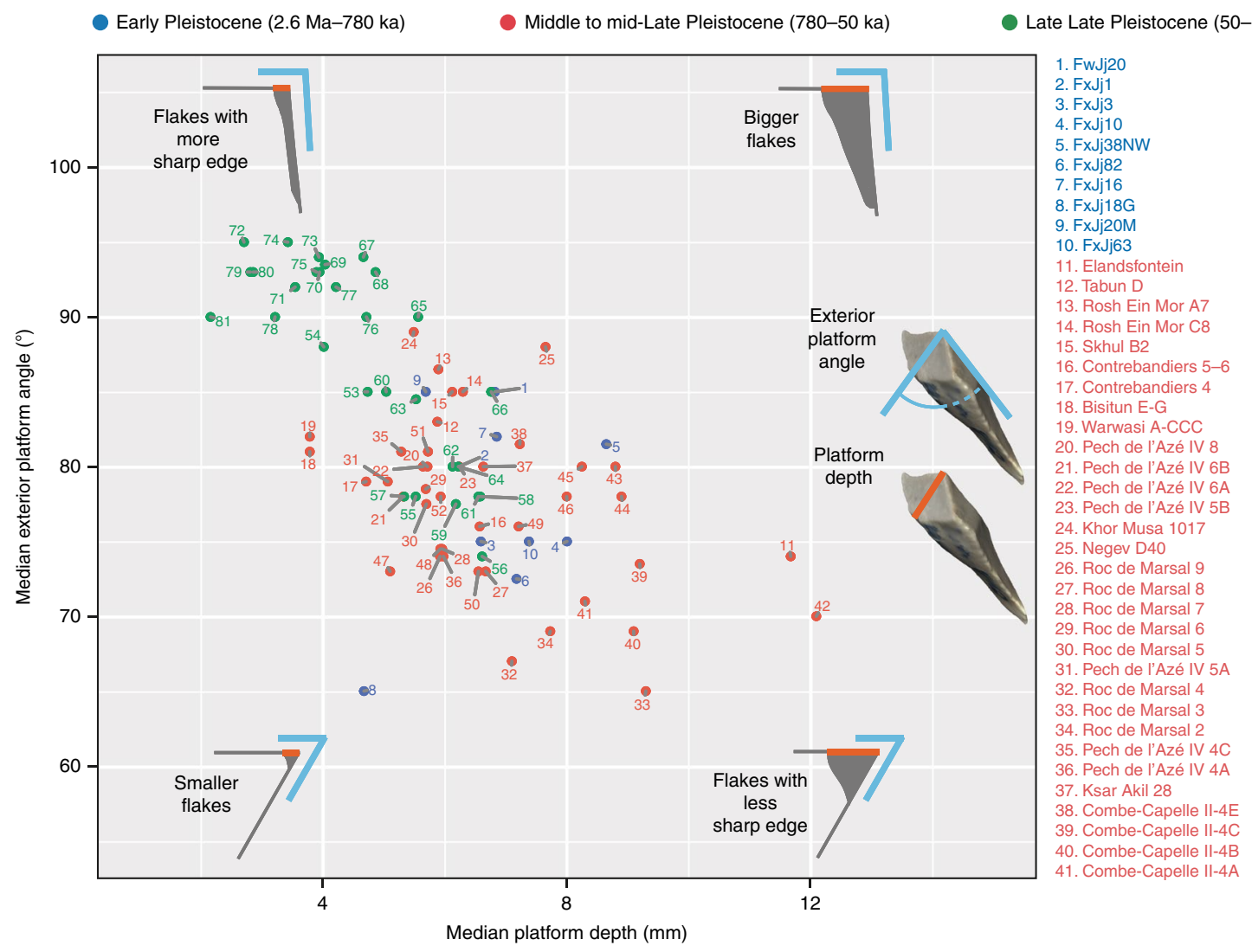

42. Combe-Capelle I-2B 43. Combe-Capelle I-2A 44. Combe-Capelle I-1E 45. Combe-Capelle I-1D 46. Combe-Capelle I-1D 47. Abri Peyrony L3A 48. Abri Peyrony L3B 49. Abri Peyrony U2 50. Abri Peyrony U3A 51. Pech de l'Azé IV 3B 52. Pech de l'Azé IV $3 A$ 53. La Côte 3

54. Roc de Combe 8 (I-K)

55. Abri Pataud 14

56. Abri Pataud 13

57. Abri Pataud 12

58. Abri Pataud 11

59. Abri Pataud 9-10

60. Boker (D100A)

61. Ein Aqev (D31) Lower

62. Ein Aqev (D31) Upper

63. Ein Aqev East (D34)

64. Abri Pataud 8

65. Abri Pataud 7

66. Abri Pataud 6

67. Abri Pataud 5

68. Abri Pataud 4

69. Abri Pataud 3

70. Abri Pataud 2

71. Laugerie Haute Est 40

72. Laugerie Haute Est 36-38

73. Laugerie Haute Est 31

74. Laugerie Haute Est 30

75. Laugerie Haute Est 21-28

76. Laugerie Haute Est 18-20

77. Laugerie Haute Est 10-16

78. Fourneau du Diable

Upper Terrace

79. Laugerie Haute Est 4-8

80. Laugerie Haute Est 2-3

81. La Madeleine 5-6

Corrected

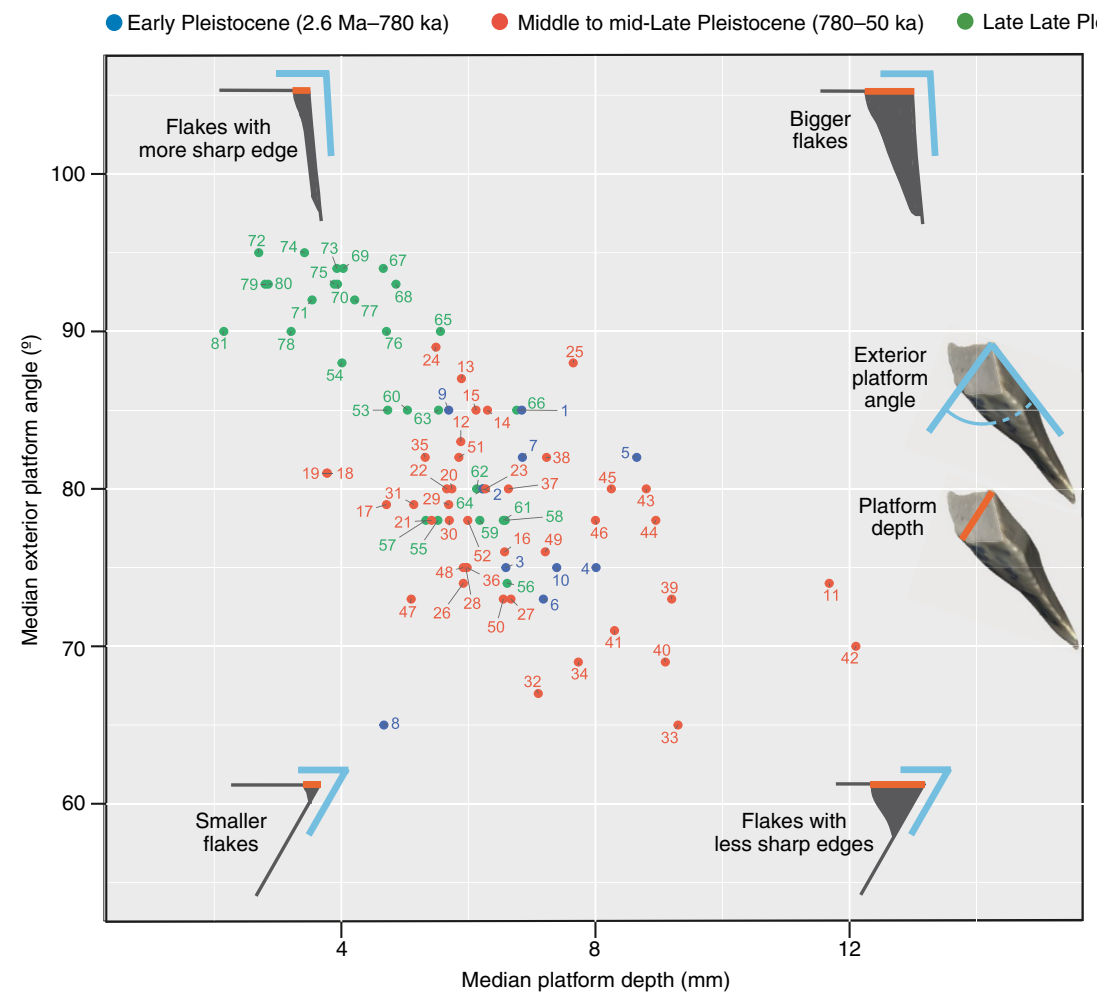

1. FwJj20

2.FXJj1

3. FxJj3

4. FxJj10

5. FxJj38NW
6. FxJj82

6. FxJj82

8. FxJj18G

9. FxJj20M

10. FxJj63

11. Elandsfontein

12. Tabun D

13. Rosh Ein Mor A7

14. Rosh Ein Mor C8

15. Skhul B2

16. Contrebandiers 5-6

17. Contrebandiers 4

18. Bisitun E-G

19. Warwasi A-CCC

20. Pech de l'Azé IV 8

21. Pech de l'Azé IV 6B

22. Pech de l'Azé IV 6A

23. Pech de l'Azé IV 5B

24. Khor Musa 1017

24. Khor Musa

26. Roc de Marsal 9

27. Roc de Marsal 8

28. Roc de Marsal 7

29. Roc de Marsal 6

30. Roc de Marsal 5

31. Pech de l'Azé IV 5A

32. Roc de Marsal 4

33. Roc de Marsal 3

34. Roc de Marsal 2

35. Pech de l'Azé IV 4C

36. Pech de l'Azé IV 4A

37. Ksar Akil 28

38. Combe-Capelle II-4E

39. Combe-Capelle II-4C

40. Combe-Capelle II-4B

41. Combe-Capelle II-4A

42. Combe-Capelle I-2B 43. Combe-Capelle I-2A 44. Combe-Capelle I-1E 45. Combe-Capelle l-1D1 46. Combe-Capelle I-1D 47. Abri Peyrony L3A 48. Abri Peyrony L3B 49. Abri Peyrony U2 50. Abri Peyrony U3A 51. Pech de l'Azé IV 3B 52. Pech de l'Azé IV $3 A$ 53. La Côte 3

54. Roc de Combe $8(\mathrm{I}-\mathrm{K})$

55. Abri Pataud 14

56. Abri Pataud 13

57. Abri Pataud 12

58. Abri Pataud 11

59. Abri Pataud 9-10

60. Boker (D100A)

61. Ein Aqev (D31) Lower

62. $\mathrm{E}$ Aqev (D31) Upe

23.

64. Abri Pataud 8

65. Abri Pataud 7

66. Abri Pataud 6

67. Abri Pataud 5

68. Abri Pataud 4

69. Abri Pataud 3

70. Abri Pataud 2

71. Laugerie Haute Est 40

72. Laugerie Haute Est 40

72. Laugerie Haute Est 36-38

73. Laugerie Haute Est 31

74. Laugerie Haute Est 30

75. Laugerie Haute Est 21-28

76. Laugerie Haute Est 18-20

77. Laugerie Haute Est 10

Fourneau du Diab

79. Laugerie Haute Est 4-8

80. Laugerie Haute Est 2-3

Median platform depth (mm)

81. La Madeleine 5-6

Fig. 1 | Original and Corrected. 

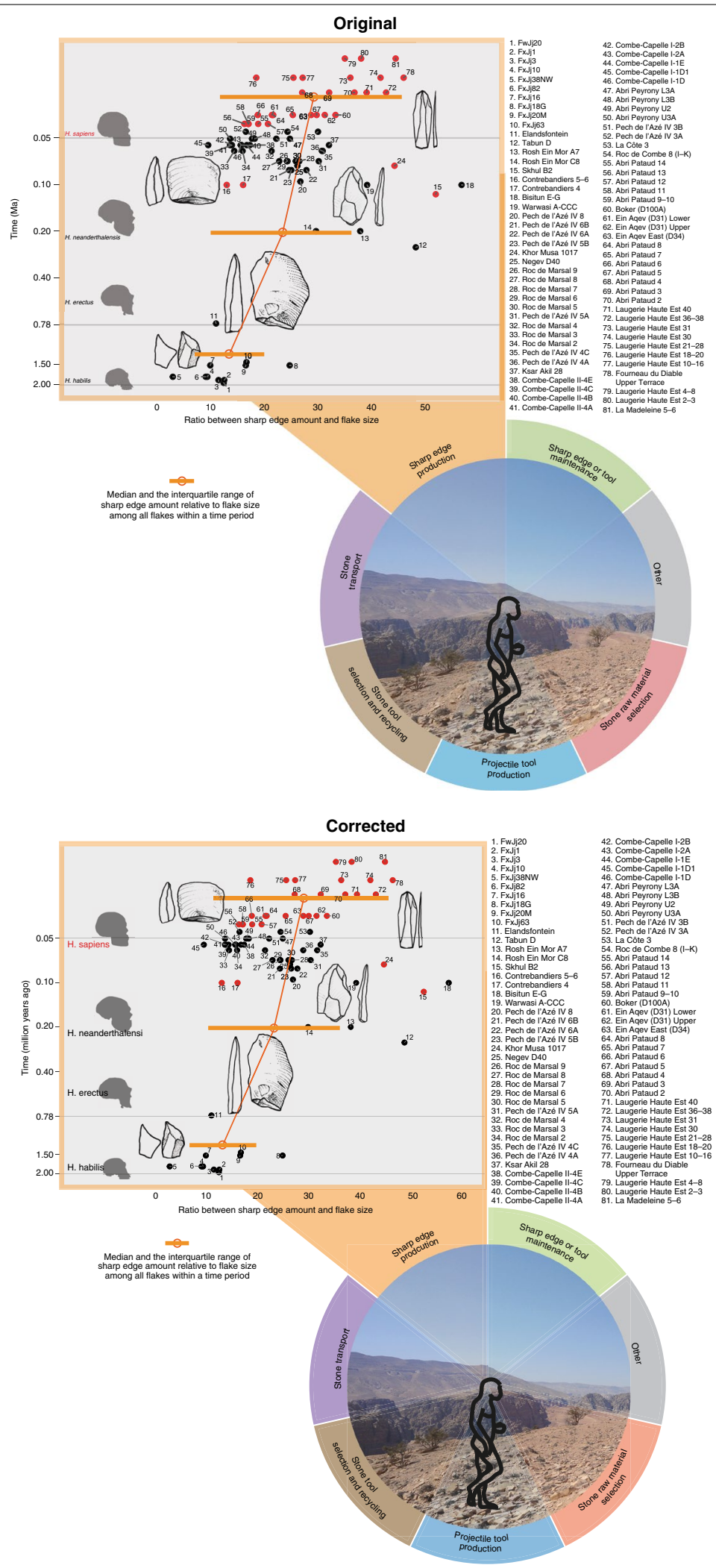

Fig. 2 | Original and Corrected. 


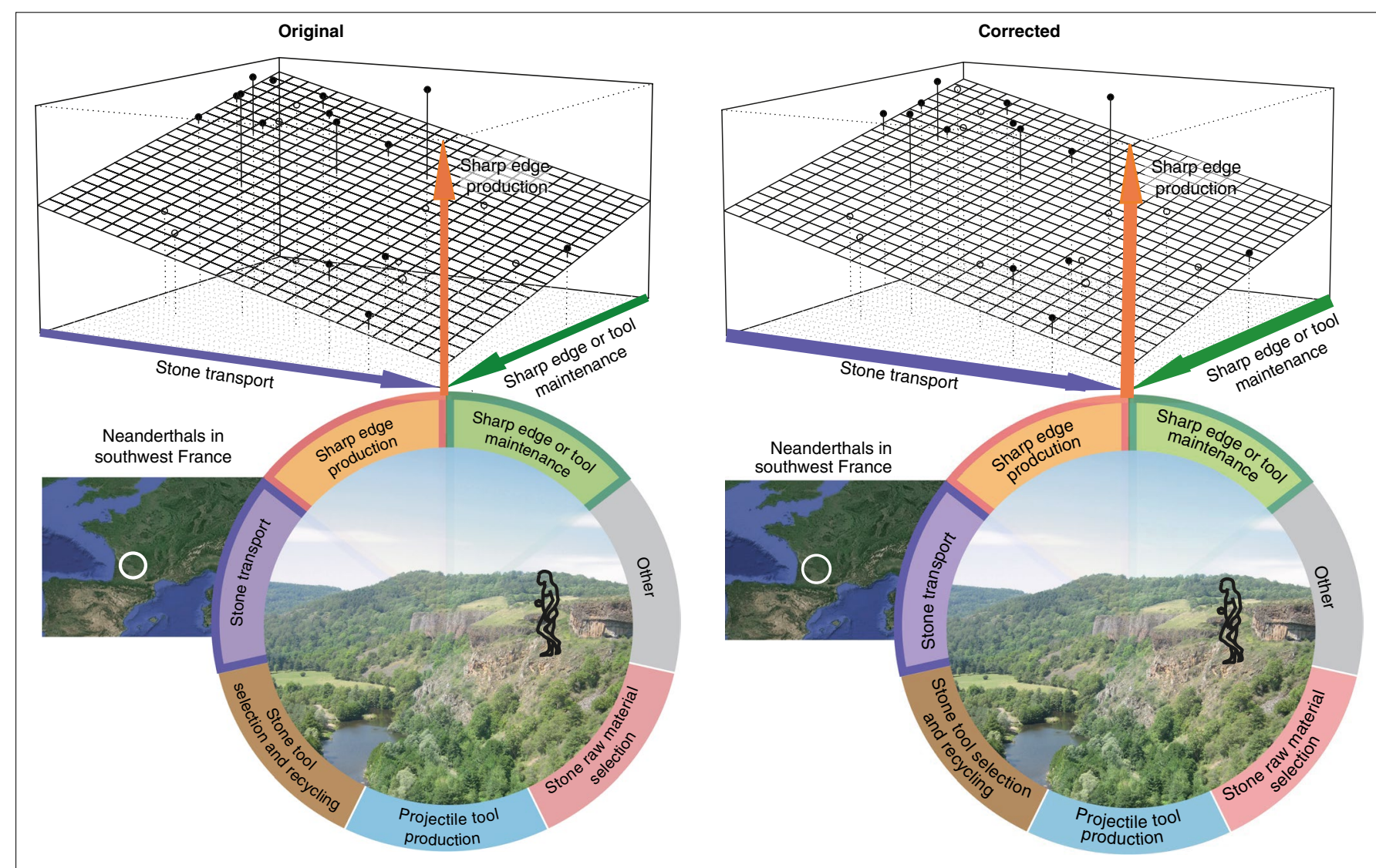

Fig. 3 | Original and Corrected. 
Original

- Early Pleistocene (2.6 Ma - $780 \mathrm{ka}) \quad$ Middle to mid-Late Pleistocene (780-50 ka) - late Late Pleistocene (50-12 ka)
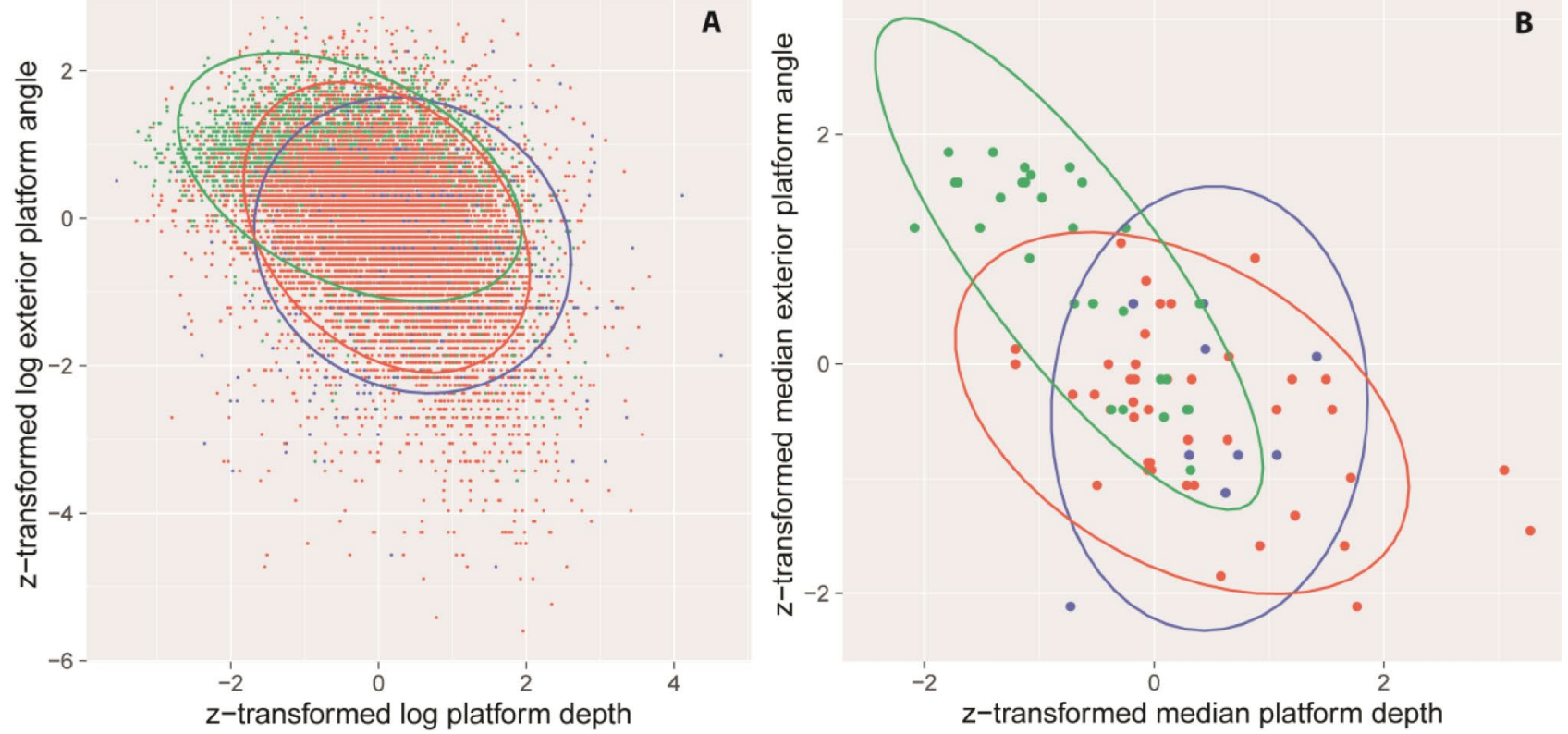

Corrected

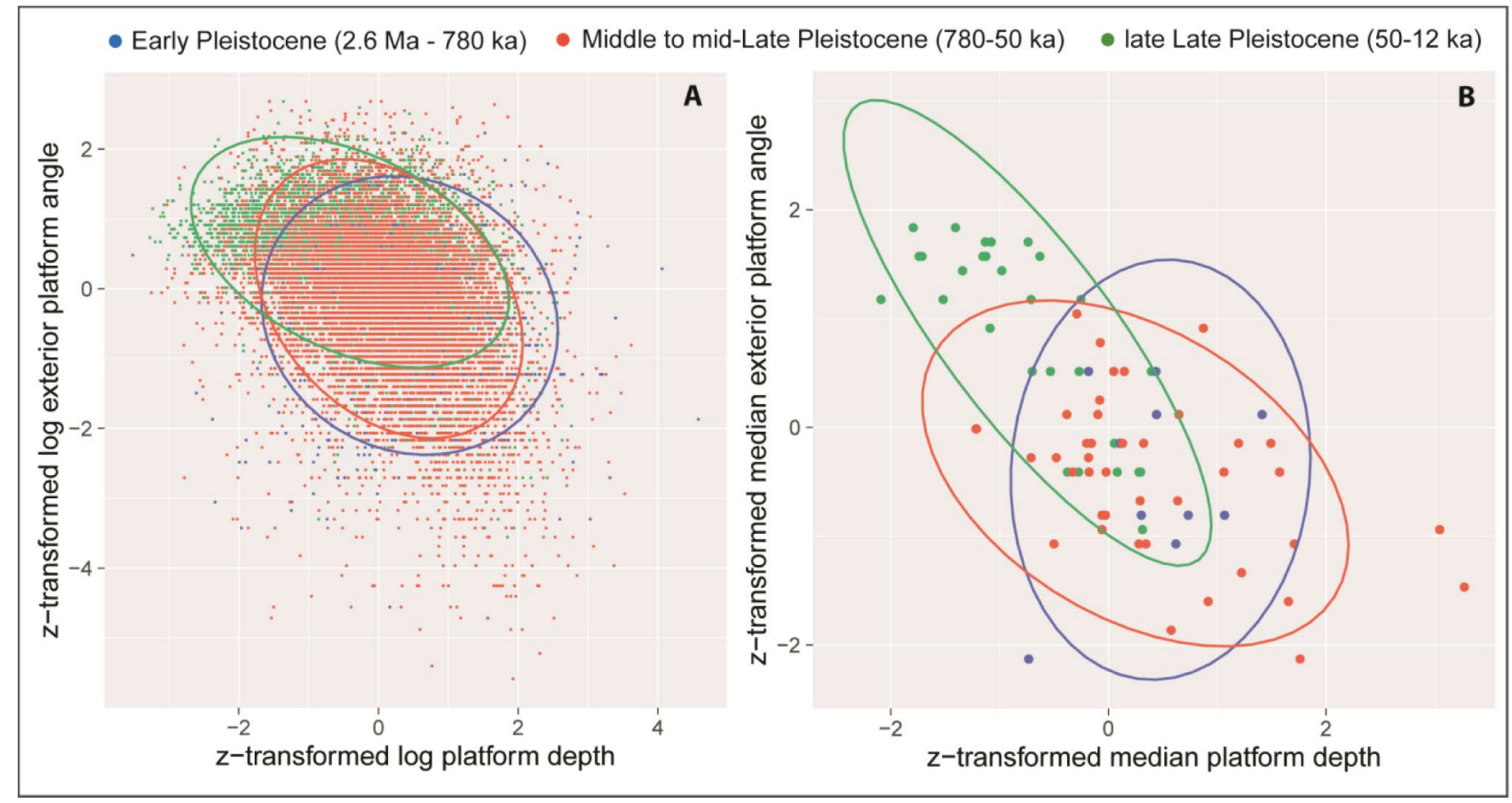

Supplementary Fig. 1 | Original and Corrected.

Published online: 25 January 2019

https://doi.org/10.1038/s41559-018-0789-7 